\title{
Corrigendum
}

\section{Subclinical zinc deficiency impairs pancreatic digestive enzyme activity and digestive capacity of weaned piglets - CORRIGENDUM}

\author{
Daniel Brugger and Wilhelm Windisch
}

(First published online 4 July 2016)

DOI: 10.1017/S0007114516002105. Published by Cambridge University Press 27 May 2016.

The authors have identified the following errors in the published article:

Tables 3 and 5: The broken line models of pancreatic chymotrypsin, carboxypeptidase a, carboxypeptidase b, and elastase activity (Table 3) as well as apparent faecal digestibility of dry matter, crude protein, total lipids and crude ash (Table 5) were based upon the linear equation: $Y=A_{X B}$ for $X \geq X_{B}$. The variable $A_{X B}$ refers to the $Y$-Intercept of the respective break-point in response. However, $A_{X B}$ should be indicated as $Y_{B}$.

Therefore, the correct linear equation is $Y=Y_{B}$ for $X \geq X_{B}$.

Table 5: The linear equations for the zinc dose range below or equal to the respective break-point in response are stated in the published article as:

$$
\begin{aligned}
& \text { Dry matter } \quad Y=484+b X \text { for } \mathrm{X} \leq X_{B} \\
& \text { Crude protein } \quad Y=-35 \cdot 4+b X \text { for } X \leq X_{B} \\
& \text { Total lipids } \quad Y=4 \cdot 22+b X \text { for } X \leq X_{\mathrm{B}} \\
& \text { Crude ash } \quad Y=212+b X \text { for } \mathrm{X} \leq X_{B}
\end{aligned}
$$

The values used in these equations should have been stated as:

$$
\begin{aligned}
& \text { Dry matter } Y=83 \cdot 0+b X \text { for } X \leq X_{\mathrm{B}} \\
& \text { Crude protein } \quad Y=77 \cdot 2+b X \text { for } X \leq X_{\mathrm{B}} \\
& \text { Total lipids } Y=50 \cdot 2+b X \text { for } X \leq X_{B} \\
& \text { Crude ash } Y=41 \cdot 0+\mathrm{bX} \text { for } X \leq \mathrm{X}_{\mathrm{B}}
\end{aligned}
$$

The correct values were used to calculate the parameter estimates and the incorrect values reported in the published articles are typographical errors only that do not affect the parameter estimates reported, or the findings and interpretation of the study.

The authors apologise for these errors.

\section{Reference}

1. Brugger D \& Windisch W (2016). Subclinical zinc deficiency impairs pancreatic digestive enzyme activity and digestive capacity of weaned piglets. Br J Nutr 116, 425-433. doi:10.1017/S0007114516002105. 


\section{The corrected tables are:}

Table 3. Broken-line regression analysis of analysed pancreatic zinc and pancreatic enzyme activity relative to dietary zinc supply (Parameter estimates with their standard errors)

\begin{tabular}{|c|c|c|c|c|c|c|}
\hline & Models & Param & estimates & SE & $P$ & $R^{2}$ \\
\hline \multirow[t]{4}{*}{ Pancreatic Zn (mg/kg DM) } & $Y=87 \cdot 2+b_{1} X$ for $X \leq X_{B}$ & $X_{B}$ & $39 \cdot 0$ & 5.09 & $<0.0001$ & 0.92 \\
\hline & $Y=56 \cdot 5+b_{2} X$ for $X \geq X_{B}$ & $Y_{B}$ & $72 \cdot 6$ & 1.39 & $<0.0001$ & \\
\hline & & $b_{1}$ & -0.38 & 0.41 & 0.39 & \\
\hline & & $b_{2}$ & 0.41 & 0.06 & $<0.0001$ & \\
\hline \multirow[t]{4}{*}{ Trypsin (U/mg per min)* } & $Y=142+b_{1} X$ for $X \leq X_{B}$ & $X_{B}$ & $58 \cdot 0$ & 8.62 & 0.0001 & 0.90 \\
\hline & $Y=39 \cdot 9+b_{2} X$ for $X>X_{B}$ & $Y_{B}$ & 172 & $10 \cdot 2$ & $<0.0001$ & \\
\hline & & $b_{1}$ & 0.52 & 0.38 & 0.21 & \\
\hline & & $b_{2}$ & 2.29 & 0.60 & 0.005 & \\
\hline \multirow[t]{3}{*}{ Chymotrypsin (U/mg per min) } & $Y=484+b_{1} X$ for $X \leq X_{B}$ & $X_{B}$ & $58 \cdot 0$ & $17 \cdot 7$ & 0.01 & 0.35 \\
\hline & $Y=Y_{B}$ for $X \geq X_{B}$ & $Y_{B}$ & 756 & $49 \cdot 8$ & $<0.0001$ & \\
\hline & $1-I_{B} \operatorname{IOH} \Lambda=\Lambda_{B}$ & $b_{1}$ & 4.69 & $3 \cdot 28$ & 0.19 & \\
\hline \multirow[t]{3}{*}{ Carboxypeptidase A (mU/mg per min) } & $Y=-35 \cdot 4+b_{1} X$ for $X \leq X_{B}$ & $X_{B}$ & $41 \cdot 2$ & $2 \cdot 74$ & $<0.0001$ & 0.82 \\
\hline & $Y=Y_{B}$ for $X \geq X_{B}$ & $Y_{B}$ & 233 & $6 \cdot 33$ & $<0.0001$ & \\
\hline & $1-1_{B} 101 A=\Lambda_{B}$ & $b_{1}$ & 6.50 & 1.87 & 0.008 & \\
\hline \multirow[t]{3}{*}{ Carboxypeptidase B (U/mg per min) } & $Y=4 \cdot 22+b_{1} X$ for $X \leq X_{B}$ & $X_{B}$ & $47 \cdot 5$ & 0.009 & $<0.0001$ & 0.63 \\
\hline & $Y=Y_{B}$ for $X>X_{B}$ & $Y_{B}$ & $6 \cdot 20$ & 0.10 & $<0.0001$ & \\
\hline & $1=Y_{B} 101 \lambda=\Lambda_{B}$ & $b_{1}$ & 0.04 & 0.01 & 0.006 & \\
\hline \multirow[t]{3}{*}{ Elastase (U/mg per min) } & $Y=212+b_{1} X$ for $X \leq X_{B}$ & $X_{B}$ & $57 \cdot 7$ & $12 \cdot 9$ & 0.002 & 0.61 \\
\hline & $Y=Y_{B}$ for $X \geq X_{B}$ & $Y_{B}$ & 326 & 9.93 & $<0.0001$ & \\
\hline & $1-I_{B} \operatorname{CON} \lambda \Lambda_{B}$ & $b_{1}$ & 1.97 & $1 \cdot 13$ & 0.12 & \\
\hline \multirow[t]{4}{*}{ a-Amylase (mU/mg per min) } & $Y=87 \cdot 1+b_{1} X$ for $X \leq X_{B}$ & $X_{B}$ & $58 \cdot 0$ & 0.03 & $<0.0001$ & 0.77 \\
\hline & $Y=64 \cdot 2+b_{2} X$ for $X \geq X_{B}$ & $Y_{B}$ & 176 & $12 \cdot 3$ & $<0.0001$ & \\
\hline & & $b_{1}$ & 1.53 & 0.67 & 0.05 & \\
\hline & & $b_{2}$ & 1.92 & 0.73 & 0.03 & \\
\hline
\end{tabular}

$R^{2}$, coefficient of determination of the respective broken-line regression model; $X_{B}, X$ intercept of the respective break point in parameter response; $Y_{B}, Y$ intercept of the respective break point in parameter response; $b_{1}$, slope of the respective broken-line regression curve over dietary $\mathrm{Zn}$ doses lesser than or equal to the respective break point in parameter response; $b_{2}$, slope of the respective broken-line regression curve over dietary $\mathrm{Zn}$ doses greater than or equal to the respective break point in parameter response.

* Pancreatic enzyme activity is expressed as units of activity change/min reaction time normalised to the total protein content within the sample; $P$ values $\leq 0.05$ were considered as indicators of statistical significance.

Table 5. Broken-line regression analysis of apparent faecal DM and crude nutrient digestibility relative to dietary zinc supply

(Parameter estimates with their standard errors)

\begin{tabular}{|c|c|c|c|c|c|c|}
\hline \multirow[b]{2}{*}{$\mathrm{DM}(\%)^{\star}$} & \multirow{2}{*}{$\begin{array}{l}\text { Models } \\
Y=83 \cdot 0+b X \text { for } X \leq X_{B}\end{array}$} & \multicolumn{2}{|c|}{ Parameter estimates } & \multirow{2}{*}{$\frac{\mathrm{SE}}{4.66}$} & \multirow{2}{*}{$\frac{P}{<0.0001}$} & \multirow{2}{*}{$\frac{R^{2}}{0.88}$} \\
\hline & & $X_{B}$ & $54 \cdot 7$ & & & \\
\hline & $Y=Y_{B}$ for $X>X_{B}$ & $Y_{B}$ & $86 \cdot 6$ & 0.13 & $<0.0001$ & \\
\hline & & $b_{1}$ & 0.07 & 0.02 & 0.003 & \\
\hline \multirow{3}{*}{ Crude protein (\%) } & $Y=77 \cdot 2+b X$ for $X \leq X_{B}$ & $X_{B}$ & $45 \cdot 0$ & 3.28 & $<0.0001$ & 0.80 \\
\hline & $Y=Y_{R}$ for $X>X_{B}$ & $Y_{B}$ & $85 \cdot 6$ & 0.28 & $<0.0001$ & \\
\hline & & $b_{1}$ & 0.18 & 0.05 & 0.006 & \\
\hline \multirow[t]{3}{*}{ Total lipids (\%) } & $Y=50 \cdot 2+b X$ for $X \leq X_{B}$ & $X_{B}$ & $46 \cdot 9$ & $2 \cdot 79$ & $<0.0001$ & 0.90 \\
\hline & $Y=Y_{B}$ for $X \geq X_{B}$ & $Y_{B}$ & $75 \cdot 7$ & 0.62 & $<0.0001$ & \\
\hline & $1-I_{B} 101 \Lambda 2 \Lambda_{B}$ & $b_{1}$ & 0.54 & 0.11 & 0.001 & \\
\hline \multirow{3}{*}{ Crude ash (\%) } & $Y=41 \cdot 0+b X$ for $X \leq X_{B}$ & $X_{B}$ & $58 \cdot 2$ & 0.03 & $<0.0001$ & 0.74 \\
\hline & $Y=Y_{B}$ for $X \geq X_{B}$ & $Y_{B}$ & $48 \cdot 2$ & 0.44 & $<0.0001$ & \\
\hline & $1-I_{B}+12-\alpha_{B}$ & $b_{1}$ & 0.12 & 0.03 & 0.002 & \\
\hline
\end{tabular}

$R^{2}$, coefficient of determination of the respective broken-line regression model; $X_{B}, X$ intercept of the respective break point in parameter response; $Y_{B}, Y$ intercept of the respective break point in parameter response; $b$, slope of the respective broken-line regression curve over dietary $\mathrm{Zn}$ doses lesser than or equal to the respective break point in parameter response.

${ }^{*}$ Coefficients of apparent faecal digestibility are expressed as percentage of feed intake; $P$ values $\leq 0.05$ were considered as indicators of statistical significance. 